\title{
Pulmonary Deposition and Clearance of Aerosolized Alpha-1-Proteinase Inhibitor Administered to Dogs and to Sheep
}

Robert M. Smith, * Lillian D. Traber, ${ }^{\star}$ Daniel L. Traber, ${ }^{\ddagger}$ and Roger G. Spragg ${ }^{\star}$

*Division of Pulmonary and Critical Care Medicine, Department of Medicine, University of California at San Diego, San Diego, California, 92103; and ${ }^{\ddagger}$ Departments of Anesthesiology and Physiology, University of Texas

Medical Branch and Shriner's Burns Institute, Galveston, Texas 77550

\begin{abstract}
Augmentation of lung antiprotease levels may be an important therapeutic intervention in the prevention of pulmonary emphysema. We have administered aerosols of plasma-derived

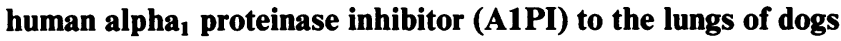
and sheep to investigate (a) delivery of the protein to the distal air spaces of the lung; (b) maintenance of functional activity of the protein; and $(c)$ flux of the protein across the components of the alveolar-capillary membrane. A1PI $(26.4 \mathrm{mg} / \mathrm{kg}$ body weight) was administered as an aerosol to anesthetized animals; sheep were prepared for the chronic collection of lung lymph. Immunoperoxidase staining of lung tissue obtained 2 h after administration of A1PI demonstrated the presence of human A1PI on the surface of alveoli and distal bronchioles. Bronchoalveolar lavage fluid recovered at intervals after A1PI administration demonstrated time-dependent elevations of human A1PI levels with augmentation of lavage fluid antielastase activity in proportion to the content of human A1PI. Using radiolabeled $A 1 P I$ as a tracer, we found that $32 \%$ of the aerosol was retained in the animals' lungs. Measurements of the rate of loss of A1PI from the lung and of the rate of appearance of human A1PI in plasma resulted in a calculated permeability of the alveolar-capillary membrane to A1PI of 3.49-6.39 $\times 10^{-10}$ $\mathrm{cm} / \mathrm{s}$. Experiments using instrumented sheep allowed independent calculation of endothelial permeability to A1PI of $122-236 \times 10^{-10} \mathrm{~cm} / \mathrm{s}$ and calculation of epithelial permeability of $4.70-4.81 \times 10^{-10} \mathrm{~cm} / \mathrm{s}$. Modeling of aerosol delivery of A1PI to humans using the results of these studies predicts that the ratio of plasma/alveolar levels of delivered A1PI will be 0.024 , and that aerosolization of $175 \mathrm{mg} \mathrm{A1PI/d}$ will result in an A1PI alveolar fluid level of $1.0 \mathrm{mg} / \mathrm{ml}$. Aerosol administration of A1PI may provide an efficient method of augmenting alveolar antiprotease levels.
\end{abstract}

\section{Introduction}

The unrestrained action of proteolytic enzymes in the lung, particularly those enzymes with elastolytic properties, may lead to the destruction of lung connective tissue and to the anatomic and functional derangements of pulmonary emphysema (1-11). In particular, neutrophil elastase has been shown

Address reprint requests to Dr. Roger G. Spragg, University of California Medical Center, 225 Dickinson Street, San Diego, CA 92103.

Received for publication 1 February 1988 and in revised form 12 April 1989.

J. Clin. Invest.

(c) The American Society for Clinical Investigation, Inc.

$0021-9738 / 89 / 10 / 1145 / 10 \quad \$ 2.00$

Volume 84, October 1989, 1145-1154 to have access to the lung and has been implicated in the development of emphysema (2-4). The dominant inhibitor of neutrophil elastase in normals, both in plasma and in bronchoalveolar lavage fluid (BAL), is alpha $a_{1}$ proteinase inhibitor $(A 1 P I)^{1}(5)$. The assumption that the presence of active A1PI in the lung is of major importance in the prevention of pulmonary emphysema is based in part on the observations that: $(a)$ patients genetically deficient in A1PI have a greatly augmented risk for developing emphysema; (b) smokers, a population with an increased incidence of emphysema, appear under some conditions to have inactive A1PI in their lavage fluid; and $(c)$ intratracheal instillation of A1PI inhibits the development of elastase-induced emphysema in some animal models. Observations such as these (reviewed in reference 1) as well as others have provided the rationale for the administration of A1PI to genetically deficient patients in an attempt to restore blood and lung antiprotease defenses.

Previous studies have focused on intravenous replacement of A1PI and have demonstrated that infusions of $\sim 60 \mathrm{mg} / \mathrm{kg}$ of active plasma-derived A1PI to PiZ patients will augment and maintain plasma and lung antielastase activity to levels predicted to protect against the development of emphysema $(12,13)$. Infusion of $83 \mathrm{mg} / \mathrm{kg}$ active A1PI into normal dogs has been shown to raise BAL A1PI activity levels by up to $74 \%$ (14). Intravenous administration of A1PI, however, is relatively inefficient in augmenting lung antielastase defenses. Measuring lung radioactivity after infusion of labeled A1PI into dogs, we have found that only $2 \%$ of the infused A1PI is resident in the lung parenchyma at equilibrium (unpublished data). Delivery of aerosolized A1PI directly to the lung via the airways may be a particularly efficient method of augmenting lung antiprotease defenses. In addition, administration of known amounts of A1PI as an aerosol provides a model with which to examine the transport of protein across the alveolarcapillary barrier.

The purpose of this study was to investigate the distribution and pharmacokinetics of A IPI delivered as an aerosol to the lungs of healthy animals. To accomplish this, we produced aerosols of A1PI which retained specific antielastase activity, and which we could demonstrate by immunohistologic studies were delivered to the alveolar level. Gamma camera imaging was used to quantify lung distribution and retention of A1PI inhaled by dogs, and lower respiratory tract secretions were assayed for the presence and activity of residual A1PI. Passage of inhaled protein into the plasma was quantified in both sheep and dogs. Using sheep prepared for the chronic collection of lung lymph, we quantified the passage of aerosolized and circulating AIPI into lung lymph to estimate interstitial

1. Abbreviations used in this paper: A1PI, alpha, ${ }_{1}$ proteinase inhibitor; BAL, bronchoalveolar lavage. 
levels of A1PI and to allow calculation of rates of protein transport across the epithelial and endothelial barriers.

\section{Methods}

AIPI preparation and assays. The A1PI used in this study (Cutter Laboratories, Berkeley, CA) was derived from Cohn fraction IV-I of human plasma (15) and subjected to heat treatment to inactivate infectious agents. The A1PI, provided in lyophilized form, was reconstituted with distilled water, diluted in an equal volume of $0.9 \%$ saline, and used within $30 \mathrm{~min}$ of reconstitution. We determined that the protein content of the lot of A1PI that was provided to us was $>80 \%$ A1PI, had predominantly PiM phenotype by acid starch gel electrophoresis, and had a specific activity of 0.74 as determined using active site titrated trypsin (16).

Aliquots of the A1PI were further purified by affinity chromatography with Con A-Sepharose (Sigma Chemical Co., St. Louis, MO) both to provide a reference standard for antigenic assays and to radiolabel for use as a tracer in animal experiments. The final product yielded a single A1PI band on 9\% SDS-PAGE and staining with Coomassie blue dye (17); the A1PI content was determined spectrophotometrically at $280 \mathrm{~nm}$ using an extinction coefficient of $5.30 \mathrm{M}^{-1} \mathrm{~cm}^{-1}$ (16). A1PI prepared in this fashion was radiolabeled with ${ }^{131} \mathrm{I}$ or ${ }^{125} \mathrm{I}$ (New England Nuclear, Boston, MA) by the solid state lactoperoxidase method (18). The resultant radiolabeled A1PI was $>98 \%$ precipitable in $10 \%$ TCA and had a specific radioactivity of $\sim 50-100 \mu \mathrm{Ci} / \mathrm{mg}$ protein. The iodinated A1PI had a specific antiprotease activity of 0.72 as determined by active-site titration with trypsin and no change relative to the starting material in ability to inhibit porcine pancreatic elastase and retained the expected electrophoretic mobility and ability to form covalent complexes with neutrophil elastase (Fig. 1). This radiolabeled A1PI was used as a tracer with the unlabeled A IPI aerosol in the canine studies to measure the amount and distribution of aerosol deposited in the lung, or was infused separately into sheep to measure the kinetics of distribution of A1PI in that model.

Antigenic content of human A1PI in plasma and lymph, as well as of canine or sheep albumin in plasma and BAL fluid, was measured by rocket immunoelectrophoresis using specific antisera (Cooper Biomedicals Inc., Malvern, PA) (19) and referenced either to the purified A1PI that we had prepared or (in later experiments) to A1PI obtained from a commercial source (Calbiochem-Behring Corp. La Jolla, CA). This latter preparation was found to be $99 \%$ pure by densitometric analysis of protein separated on a $9 \%$ SDS polyacrylamide gel and stained with Coomassie blue. Levels of human A1PI in lung lavage fluid were measured either by rocket immunoelectrophoresis or, for samples $<2 \mu \mathrm{g} \mathrm{A1PI/ml,} \mathrm{by} \mathrm{ELISA} \mathrm{as} \mathrm{reported} \mathrm{previously} \mathrm{(14).} \mathrm{There}$ was no cross-reactivity in either antigenic assay to dog or sheep plasma.

Measurement of AIPI is confused by the overestimation of the true A1PI content in commercially available standards and consequently, overestimation of what constitutes a normal level in many widely quoted sources $(13,16,20)$. When the purified A1PI that was used as a reference in our antigenic assays was compared by radial immunodiffusion to a commercial standard (Behring Diagnostics, Hoechst, Inc., Somerville, NJ), the content of A1PI in the commercial standard was overestimated by $49 \%$. If conversion of the values reported in this paper to those derived using such a commercial standard is desired, our values for A1PI content should be multiplied by 1.49 .

The elastase inhibitory capacity of BAL samples was determined by measuring their ability to inhibit the hydrolysis of succinyl-(alanyl) $)_{3}-p-$ nitroanilide (Sigma Chemical Co) by porcine pancreatic elastase (Elastin Products Co., Pacific, MO) (21). Activity was referenced to the commercial A1PI standard (Calbiochem-Behring Corp.) that we determined to have a specific activity of 0.90 using active-site titrated trypsin (16).

To determine whether aerosolized human AIPI recovered in BAL was intact, cleaved, or complexed to enzyme, BAL samples from seven dogs were subjected to $10 \%$ SDS-PAGE (17), blotted onto nitrocellulose, and exposed to horseradish peroxidase- (HRP) conjugated goat anti-AlPI (Organon Teknika Cappel, West Chester, PA). Antibody localization was visualized using 4-choro-1-napthol and $\mathrm{H}_{2} \mathrm{O}_{2}$.

Aerosol production. We aerosolized the A1PI preparation using an ultrasonic nebulizer (Mistogen model EN143A; Oakland, CA). Particle size was measured by Dr. R. Mannix and Dr. R. Phalen of the University of California, Irvine. A seven-stage cascade impactor (22) was used to sample the ${ }^{131} \mathrm{I}-\mathrm{A} 1 \mathrm{PI}$ aerosol. The measured activity (mass) median aerodynamic diameter was $5.1 \mu \mathrm{m} \pm 1.9 \mathrm{GSD}$.

The ability of the A1PI to retain its antielastase activity during ultrasonic nebulization was tested in preliminary experiments in which precipitated particles in downstream tubing and residual material in the nebulizer chamber were sampled at 5-min intervals. The specific antielastase activity of samples was compared with that of the starting material, and as previously reported, neither the recovered material nor that remaining in the nebulizer chamber showed a significant change over a 40 -min period (23).

All aerosols were delivered while the animals were anesthetized ( 25 $\mathrm{mg} / \mathrm{kg}$ pentobarbital for dogs, $2 \%$ halothane for sheep), restrained (dogs were supine, sheep were prone), and mechanically ventilated (15 $\mathrm{ml} / \mathrm{kg}, 12$ breaths $/ \mathrm{min}, \mathrm{F}_{\mathrm{i}} \mathrm{O}_{2}=0.21$ ). In all animals, $26.4 \mathrm{mg} / \mathrm{kg}$ of A1PI in a volume of $1.4 \mathrm{ml} / \mathrm{kg}$ and, in the dog studies, an additional $150 \mu \mathrm{Ci}$ of purified ${ }^{131} \mathrm{I}$-A $1 \mathrm{PI}$ was aerosolized over $30-45 \mathrm{~min}$ into the inspiratory limb of the ventilation circuit. Serial downstream filters (nonconductive Anesthesia Filter; American Hospital Supply, McGraw, IL) were used to capture exhaled particles when radiolabeled

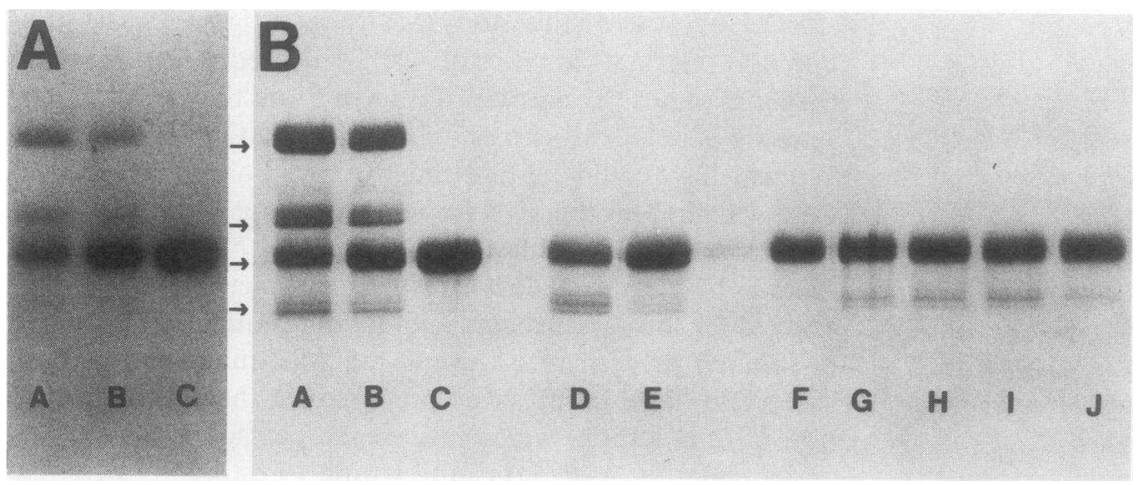

Figure 1. A1PI containing $2 \%{ }^{125}$ I-A IPI was allowed to react with neutrophil elastase at $23^{\circ} \mathrm{C}$ for $15 \mathrm{~min}$. Aliquots of the reaction mixtures and samples of canine BAL were then subjected to gel electrophoresis and Western blotting $(B)$ and an autoradiograph of the blot was obtained $(A)$. (Arrows) Bands of molecular mass 76, 71, 52, and $47 \mathrm{kD}$. Lanes $A$, $B$, and $C$ were each loaded with $10 \mu \mathrm{g} \mathrm{A1PI}$ incubated with $5,2.5$, or $0 \mu \mathrm{g}$ human neutrophil elastase (Elastin Products Co., Pacific, $\mathrm{MO}$ ), respectively. Lanes $D$ and $E$ contain samples from two dogs in group II; lanes $F-J$ contain samples from five dogs in group I. $A$ demonstrates that ${ }^{125} \mathrm{I}-\mathrm{A} 1 \mathrm{PI}$ is capable of

forming complex with enzyme and yielding the four expected products (complex, complex containing cleaved A1PI, A1PI, and cleaved A1PI). $B$ demonstrates that human A1PI recovered in canine BAL was not complexed to enzyme and showed evidence of only minimal cleavage to the $47-\mathrm{kD}$ fragment. 
A1PI was used; all exhaled radioactivity was contained in the first of two filters.

Immunohistologic studies. Specimens of lung for immunohistologic staining were obtained $2 \mathrm{~h}$ after aerosol administration in two dogs. After administration of A1PI, a balloon-tipped pulmonary artery catheter was introduced and advanced under fluoroscopic guidance to a distal pulmonary artery branch. At the time of killing, the balloon was inflated to isolate the vasculature of the distal lung, and the animal was euthanized with a bolus of pentobarbital. The lungs were statically inflated with air $(30 \mathrm{ml} / \mathrm{kg})$ and the isolated segment of lung was perfused with $60 \mathrm{ml}$ of $3 \%$ dextran followed by $100 \mathrm{ml}$ of fixative containing $2 \%$ paraformaldehyde, $0.2 \%$ picric acid, and $0.2 \%$ glutaraldehyde. $5 \mathrm{~min}$ after introduction of the fixative, the animals' chests were opened and the perfused and fixed volume of lung was removed. Samples obtained in an identical fashion from dogs not exposed to human A1PI were used as negative controls. The fixed specimens were embedded in paraffin and thin sections were examined by immunoperoxidase staining for the presence of human A1PI using rabbit antihuman A1PI (Dako Corp., Santa Barbara, CA) and an immunoperoxidase staining kit (Vector Laboratories, Burlingame, CA) (24). The anti-human A1PI antibody did not react by immunodiffusion assay with dog or sheep plasma, and gave a single precipitin line when reacted with the unpurified human A1PI preparation.

Radioactivity assays. Radioactivity of tissue, blood, and lung samples was measured in a gamma well counter (Searle Analytics, Des Plaines, IL) and corrected for radioactive decay. Radioactivity of the supernatants of a $10 \%$ TCA precipitation of parallel samples was also measured. Whole body gamma camera scans were obtained in dogs (Picker Systems, Inc., New York), digitized in a $64 \times 64$ pixel array (Medical Data Systems, Ann Arbor, MI) and stored. The response of the gamma camera to ${ }^{131} I$ was measured using both point $\left(0.64 \mathrm{~cm}^{2}\right)$ and diffuse $\left(218 \mathrm{~cm}^{2}\right)$ sources of radioactivity, and was shown to be linear $(r>0.997)$ over the range of activity encountered in the study. Sequential 5-min scans were obtained while the animals were anesthetized, mechanically ventilated, and placed supine at a fixed distance from the camera. At the conclusion of each study, the stored images were retrieved and regions of interest (ROIs) were chosen over the centers of each lung, the heart, the liver, and the spleen. For each ROI, a plot of counts per 10 pixels vs. time was generated and corrected for radioactive decay. The nebulizer chambers containing the radiolabeled A1PI were counted under the gamma camera before aerosol administration. The chamber, ventilator tubing, endotracheal tube, and downstream filter were counted in an identical fashion following aerosol delivery and the counts subtracted from the initial value to quantify the fraction of aerosolized protein retained in each animal.

Bronchoalveolar lavage. Dogs were anesthetized, intubated, and mechanically ventilated as described. A cuffed $5.2-\mathrm{mm}$ flexible fiber optic bronchoscope (Machida America Inc., Norwood, NJ) was inserted into a subsegment of the right middle lobe or lingula, the cuff was inflated, and five $30-\mathrm{ml}$ lavages of sterile $0.9 \% \mathrm{NaCl}$ were performed. Each recovered aliquot was filtered through a single layer of gauze and centrifuged at $500 \mathrm{~g}$ for $10 \mathrm{~min}$. The supernatant was removed, the cell pellet was resuspended, $1 \times 10^{5}$ cells were used to make a cytocentrifuge smear (Cytospin; Shandon Southern, Inc., Runcorn, Cheshire, England), and a differential count of 200 cells was obtained. Aliquots of the supernatant were analyzed for ${ }^{131} I$ radioactivity with and without TCA precipitation, and the remainder was stored at $-70^{\circ} \mathrm{C}$. All assays were performed on all five aliquots from each individual animal; when normalized to BAL canine albumin concentration, there were no significant differences between values in successive aliquots, and therefore all lavage data are expressed as if obtained in a single pooled lavage.

Preparation of sheep for collection of lung lymph. Ewes of Suffolk or Merino breed were prepared in two separate operations by a modification of the technique of Staub et al. (25). In the first operation, a silicone rubber catheter was placed in the left atrium through a thoracotomy in the left fifth intercostal space. During this procedure, the borders of the diaphragm and posterior aspect of the left thoracic cavity were cauterized to sever systemic afferent lymphatics that might enter the caudal mediastinal lymph node. 1 wk later, a right thoracotomy was performed through the sixth intercostal space. The caudal mediastinal node was isolated, and a single efferent vessel of the node was cannulated. Any other efferents encountered were ligated. A second incision was made in the ninth intercostal space and the distal end of the caudal mediastinal node was ligated. The border of the diaphragm and the posterior aspects of the right hemithorax were cauterized to prevent systemic contamination of the efferent lymph flow.

In all sheep, a flow-directed thermal-dilution Swan-Ganz catheter (model 93-A-131-7F; American Edwards Laboratories, Santa Ana, CA) was positioned in the pulmonary artery. At the same time, an arterial catheter was placed in the femoral artery and advanced into the thoracic aorta. These catheters were tunneled from the femoral region under the skin and brought up to the surface of the flank. All surgical procedures were performed under halothane anesthesia.

Experimental protocols. In the first series of experiments, 15 healthy mongrel dogs weighing $17.9 \pm 2.5 \mathrm{~kg}$ were studied. Each animal was shown to have normal arterial blood gases; WBC, RBC, and platelet count; plasma blood urea nitrogen, creatinine, and albumin concentrations; and chest radiographs. To control for the potential effects of BAL on subsequent clearance of A1PI from the airway three groups of dogs were studied. Dogs were lavaged only once at 6 (group I), 24 (group II), or $144 \mathrm{~h}$ (group III) after administration of A1PI aerosol (Table I).

Arterial blood gases were sampled before and at the completion of aerosol inhalation. Additional blood samples were obtained before, 15 min after the start, and at the end of aerosol inhalation for red cell count, platelet count, and for the leukocyte count and differential. Venous blood samples were collected into acid-citrate-dextrose-containing tubes at the completion of aerosol inhalation, at 30-min intervals for $2 \mathrm{~h}$, hourly for $6 \mathrm{~h}$, and then daily until the time of killing. Plasma was separated and stored at $-70^{\circ} \mathrm{C}$. Gamma camera scans were obtained as described above for the first $6 \mathrm{~h}$ after inhalation, then daily until time of killing.

All dogs received an overdose of pentobarbital immediately after BAL. The lungs, liver, spleen, kidneys, thyroid, and gonads were removed, weighed, and multiple $1.0-\mathrm{g}$ portions were removed for assay of ${ }^{131}$ I radioactivity. Specimens from the lungs were obtained from the periphery and care was taken not to include tissue from large or medium-sized airways.

Our initial results suggested that measurement of A1PI concentration in the pulmonary interstitium would be of value in defining the permeability of the alveolar-capillary membrane to A1PI, and for this reason a second series of experiments was performed in which aerosols of A I PI (without radioactive A1PI) were administered to instrumented and anesthetized sheep that weighed $52 \pm 9.2 \mathrm{~kg}$. Baseline half-hour lymph collections with concurrent plasma samples were obtained $24 \mathrm{~h}$ before and over the hour immediately preceding A1PI administration. After A1PI administration, collections of lymph over $30 \mathrm{~min}$ were obtained hourly for $8 \mathrm{~h}$, and two sequential half-hour collections were

Table I. Experimental Groups in Canine Studies

\begin{tabular}{lccc}
\hline Group & I & II & III \\
\hline $\begin{array}{l}\text { Number of animals in group } \\
\text { Time of lavage and organ collection } \\
\text { after aerosol delivery }(h)\end{array}$ & 5 & 6 & 4 \\
$\begin{array}{l}\text { Retention of aerosolized }{ }^{131} \text { I A 1 PI } \\
\text { in lung (\%) }\end{array}$ & 6 & 24 & 144 \\
\hline
\end{tabular}

The 15 animals were distributed into three groups. The amount of ${ }^{131}$ I-A1PI retained in the chest of each animal was obtained by subtracting the amount of radioactivity present in the aerosolizing circuit after aerosolizing from the amount initially placed in the nebulizer chamber. Results are expressed as mean \pm SD. 
obtained daily for $7 \mathrm{~d}$; blood samples were obtained every $30 \mathrm{~min}$ for 8 $\mathrm{h}$, and at the beginning and end of the daily lymph collections following A1PI administration.

To combine data from the experiments described here and from our prior experience (14), thereby allowing modeling of protein clearance from the lower airway, it is necessary to show that the pharmacokinetics of intravenously administered A1PI are similar in sheep and dogs. Therefore, $100 \mu \mathrm{Ci}$ of ${ }^{125} \mathrm{I}-\mathrm{A} 1 \mathrm{PI}$, protein content $<0.2 \mathrm{mg}$, was administered as an intravenous bolus to the same four sheep that had received aerosolized A1PI. The lymph and plasma collections were assayed for radioactivity, before and after precipitation in 10\% TCA, and the results analyzed for the calculation of pharmacokinetic parameters. Thus, antigenic assays for human A1PI were used to assess transport of aerosolized A1PI from the lung into lymph and plasma, and radioactivity assays were used to measure the pharmacokinetics of intravenously administered A1PI. Animal studies described were approved in advance by the institutional review boards and radiation safety committees at the University of California, San Diego.

Statistical methods. Data are presented as mean values \pm SD. Group values were compared by one way analysis of variance and, when a statistically significant difference was found, individual groups were compared using a two-tailed $t$ test with a Bonferoni correction (26). Differences between groups were felt to be significant when $P<0.05$.

The values for counts in the lung ROIs over time were fit to a biexponential equation using curve stripping (27) and nonlinear least squares regression (28) performed on a microcomputer. Values for plasma radioactivity in sheep were analyzed in a similar fashion and the final estimates for the parameters of the biexponential equation were used to calculate pharmacokinetic parameters, including halflives and volumes of distribution (29).

\section{Results}

Gamma camera scanning. At the conclusion of aerosol delivery to dogs, the animals were extubated and the aerosol chamber, ventilation tubing, endotracheal tube, and downstream filters were counted. Of the ${ }^{131} \mathrm{I}-\mathrm{A} 1 \mathrm{PI}$ initially placed in the aerosol chamber, $32.4 \pm 9.9 \%$ was retained in each animal. The appearance of the scans immediately after aerosol administration demonstrated a homogeneous distribution of counts over the lung fields. The presence of a centrally located "hot spot," suggesting pooling of the radiolabel in one of the central airways, was noted in one lung of 4 of the 15 animals. When this was seen, the involved lung was excluded from the whole lung ROI and only the contralateral lung was scanned. The counts per 10 pixels obtained during the first $6 \mathrm{~h}$ after aerosol administration in ROIs chosen over the innermost, middle, or outermost thirds of the lung fields were analyzed in three animals that did not demonstrate this central pooling. There was an $18.4 \pm 4.5 \%$ decrease in the counts over the innermost lung fields compared with $9.5 \pm 4.7$ and $4.9 \pm 5.7 \%$ decreases in the middle and outer thirds, respectively (Fig. 2). The rate of decrease in the counts over the innermost third was significantly greater $(P<0.05)$ than in the middle and outer thirds.

Counts from whole lung ROIs from all scans in the dogs studied for $144 \mathrm{~h}$ were displayed on a semilog plot (Fig. 3) and fitted to a biexponential model. The correlation coefficient for the fit of the ROI data from each individual dog was $>0.99$. ROI activity decreased with an initial $t_{1 / 2}$ of $12.3 \pm 3.14 \mathrm{~h}$ and then slowed to a late phase $t_{1 / 2}$ of $50.8 \pm 8.8 \mathrm{~h}$. The component of the lung ROI counts that was cleared with a slower $t_{1 / 2}$ was $50.5 \pm 35 \%$ of the initial lung ROI counts, suggesting that half of the initially aerosolized material was deposited in a site where it was subjected to a more rapid clearance. This estimate is in agreement with values for central versus peripheral depo-

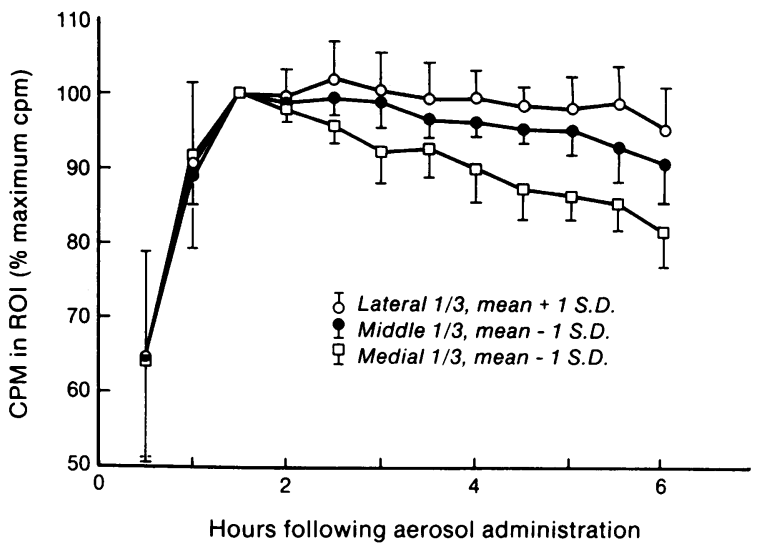

Figure 2. Radioactivity measured by external gamma camera scanning from concentric ROIs over the most medial, middle, and most lateral thirds of each lung is plotted against the time after onset of aerosol delivery. Data are expressed as a percent of counts in the $\mathrm{ROI}$ at $1.5 \mathrm{~h}$. The decrease in radioactivity measured over the central areas of each lung is most rapid $(P<0.05)$.

sition reported for similarly sized particles in dogs (30), and suggests that $16 \%(50.5 \% \cdot 32 \%)$ of the initially aerosolized A 1PI was deposited into the lung periphery. Finally, the presence of a biexponential clearance is consistent with our observation of more rapid clearance of A1PI from central lung regions, the dominant site of larger airways.

Immunohistologic studies. Examination of specimens obtained $2 \mathrm{~h}$ after aerosol administration of A1PI demonstrated specific staining for the presence of human A1PI on the lumenal surface of alveoli (Fig. 4). In addition, there was intense staining of the surface of bronchioles, and small aggregates of amorphous material were seen adherent to the walls of larger airways. The specimens showed staining of all alveolar surfaces, though there was some inhomogeneity in the intensity. Specific staining was also apparent in the interstitium, consistent with early passage of the A1PI across the alveolar epithelial membrane. Similarly prepared specimens of lung from dogs not exposed to human A1PI showed none of this specific staining.

Blood gas and hematologic values after aerosolization. There was no change in $\mathrm{P}_{a} \mathrm{O}_{2}, \mathrm{p}_{a} \mathrm{H}$, or $\mathrm{P}_{a} \mathrm{CO}_{2}$ between samples of arterial blood obtained before aerosol administration and those obtained at its completion. Similarly, there was no significant difference in RBC count, platelet count, or WBC count

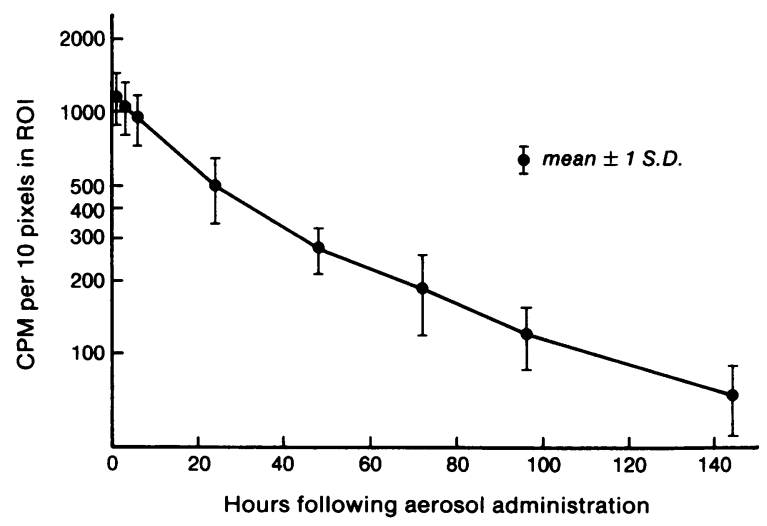

Figure 3. Radioactivity in a whole lung ROI in group III dogs is plotted against the time following administration of A1PI aerosol. 

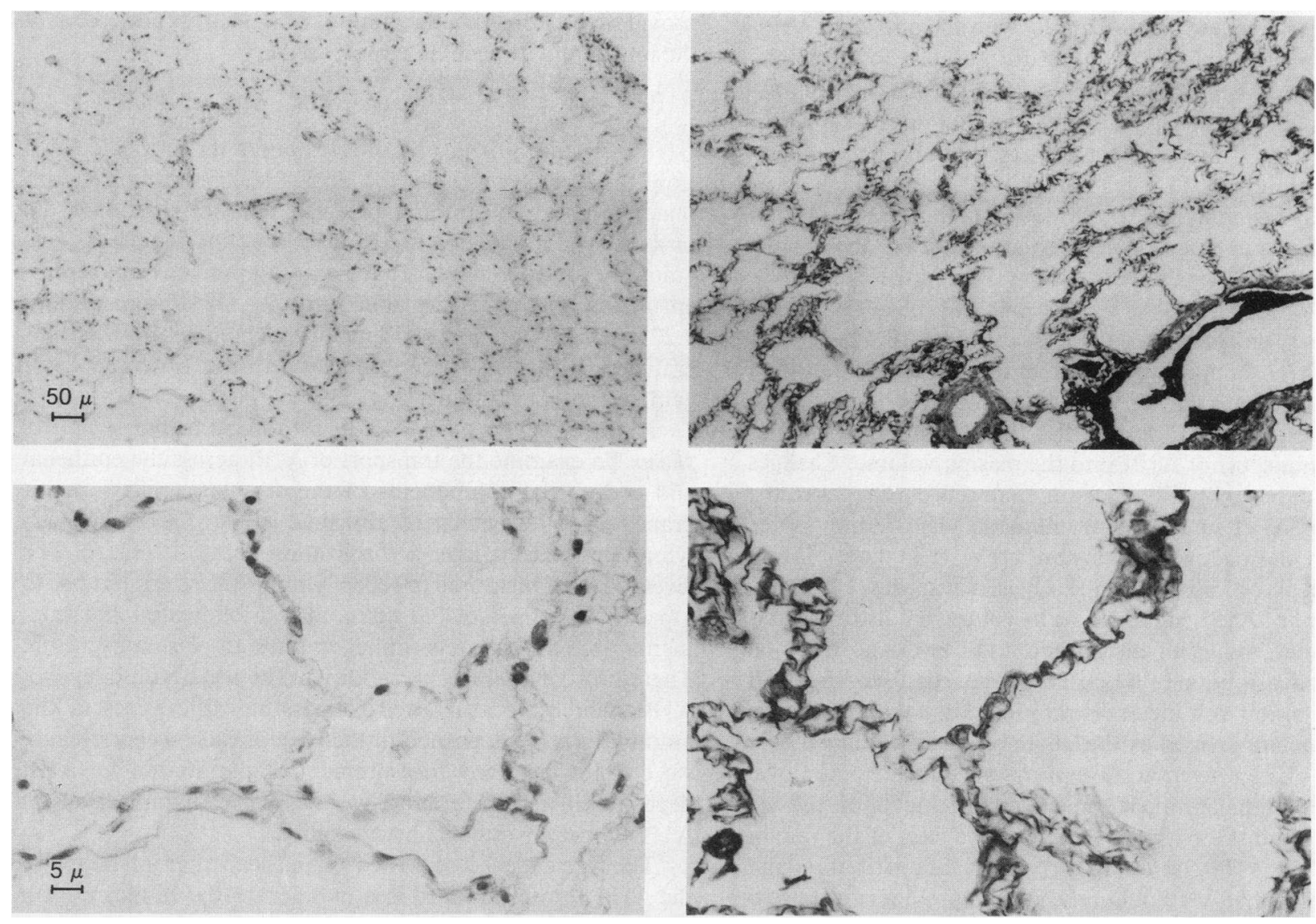

Figure 4. Specimens from the periphery of canine lung were obtained after in situ vascular perfusion fixation (see text) and stained for the presence of human A1PI. The sections are shown at an initial magnification of 40 (top) and an initial magnification of 400 (bottom). (Right) Obtained from an animal $2 \mathrm{~h}$ after inhalation of $26.4 \mathrm{mg} / \mathrm{kg}$ of human A1PI aerosol and show black-specific staining of the lumenal surface of alveoli and bronchi. Those on the left, stained in an identical fashion, were obtained from an animal never exposed to human A1PI.

and differential, between samples obtained before, during, or after aerosol A1PI administration.

AlPI content and antielastase activity of BAL. BAL volume, albumin content, number of cells, and cell differential from dogs in groups I, II, and III are shown in Table II; no significant differences were found between groups. BAL human A1PI content, normalized to canine albumin, was $911 \pm 449,135 \pm 56$, and $23 \pm 9 \mu \mathrm{g} \mathrm{A1PI} / \mathrm{mg}$ albumin in the respective groups lavaged at 6,24 , and $72 \mathrm{~h}$ after aerosol administration of A1PI. All values were significantly different from each other, $P \leq 0.05$.

To investigate the state of A1PI recovered in BAL, samples were subjected to SDS-PAGE and Western blotting. Results of all samples analyzed (Fig. 1) indicate that A1PI in BAL was intact and migrated as a $52-\mathrm{kD}$ protein. Only a minor fraction showed evidence of proteolytic cleavage to the $47-\mathrm{kD}$ product, a cleavage that may have resulted from interaction with canine protease(s). No evidence of complex formation with protease was found. The specific antielastase activity of AIPI recovered in all canine BAL samples (referenced to active-site titrated A1PI) was estimated after subtracting from the measured activity an assumed native canine BAL antielastase activity of $21.2 \mu \mathrm{g} \mathrm{A} 1$ PI equivalents/mg albumin (14). The resultant average specific activity was $0.56 \pm 0.08$ and was not different between groups.

Passage of aerosolized AIPI into plasma of dogs. Plasma levels of antigenic A1PI in group III dogs rose slowly to a maximum value at $48 \mathrm{~h}$ after administration, remained ele- vated from $48-72 \mathrm{~h}$, and then declined slowly by $144 \mathrm{~h}$ after administration (Fig. 5). The pattern of the slow rise to $48 \mathrm{~h}$, plateau, and then gradual decline was seen in each of the animals, despite the variability in the relative levels between animals. We elected not to correct the plasma levels by the amount of the A1PI that was retained in each animals chest, but instead used the uncorrected plasma levels of A1PI to calculate the transport rates for A1PI out of the lung. Plasma

Table II. Dog Lavage Data

\begin{tabular}{lccc}
\hline \multicolumn{1}{c}{ Group } & I & II & III \\
\hline $\begin{array}{l}\text { Volume recovered }(\mathrm{ml}) \\
(\% \text { instilled volume })\end{array}$ & $114 \pm 4$ & $119 \pm 7$ & $119 \pm 7$ \\
$\begin{array}{l}\text { Albumin recovered } \\
(\mu \mathrm{g} / \text { lavage })\end{array}$ & $4953 \pm 3207$ & $5803 \pm 1502$ & $2611 \pm 363$ \\
Leukocytes & & & \\
$\quad\left(\right.$ cells $\times 10^{7} /$ lavage $)$ & $4.62 \pm 3.90$ & $3.14 \pm 3.08$ & $3.83 \pm 2.62$ \\
Differential $(\%)$ & & & \\
Macrophage & & & \\
Lymphocyte & $98 \pm 1$ & $98 \pm 2$ & $93 \pm 6$ \\
PMN & $1 \pm 1$ & $1 \pm 1$ & $4 \pm 3$ \\
Epithelial & $1 \pm 1$ & $1 \pm 1$ & $4 \pm 3$ \\
& $0 \pm 0$ & $0 \pm 0$ & $0 \pm 0$ \\
\hline
\end{tabular}

The results of assays performed on canine lavage fluid are expressed as the mean $\pm \mathrm{SD}$ for the pooled lavage fluid. No significant differences in the results of these assays were found between groups. 
radioactivity rose and fell in parallel with the antigenic A1PI, and similar fractions of the inhaled dose were found in the plasma using either radioactivity or antigenic assays, supporting the validity of the use of using the radiolabeled A1PI in gamma camera scans to reflect pulmonary transport.

Levels of human A1PI per milliliter of plasma were too low to permit assessment of the contribution of the inhaled protein to total plasma antielastase activity, and therefore the specific antielastase activity of the human A1PI could not be measured.

Values for plasma A1PI clearance $(0.751 \pm 0.154$ $\mathrm{ml} \cdot \mathrm{kg}^{-1} \cdot \mathrm{h}^{-1}$ ) (unpublished data) and steady state volume of distribution $(99.9 \pm 21.0 \mathrm{ml} / \mathrm{kg})(14)$ in dogs were used with the measured plasma A1PI levels after aerosol administration to calculate transport of A1PI into the plasma volume. Changes in plasma levels of A1PI are assumed to reflect changes in the content of A1PI in its usual volume of distribution, since changes in plasma levels occur slowly relative to the equilibration rate between vascular and extravascular spaces (14). Thus, the amount of A1PI contained in its volume of distribution is equal to the product of the plasma A1PI level and the total volume of distribution of A1PI. The rate of change of the A1PI contained in this volume of distribution is equal to the change in A1PI content divided by the length of the time interval over which the change occurred. The simultaneous rate of removal of A1PI out of the plasma is equal to the product of the plasma A1PI level and the plasma clearance. The sum of the rate of change of the A1PI content in the volume of distribution and rate of removal of A1PI out of the plasma provides an approximation of total A1PI transport into the plasma at any given time after aerosolization. If there is no other pathway for A1PI movement into the plasma except out of the lung, then this value provides an estimate of the rate of A1PI transport out of the lung.

The plasma levels of A1PI in the group III dogs were used to calculate A1PI transport out of the lung in accord with this analysis (Fig. 5, inset). The transport rates were used to calculate the monoexponential equation:

$\frac{\mathrm{d}[\mathrm{A} 1 \mathrm{PI}]}{\mathrm{d} t}=0.1021 \mathrm{e}^{-0.0252 t} \mathrm{mg} \cdot \mathrm{kg}^{-1} \cdot \mathrm{h}^{-1} \quad(r=0.98)$

The integral of this equation (from time $=0$ to time $=t$ )

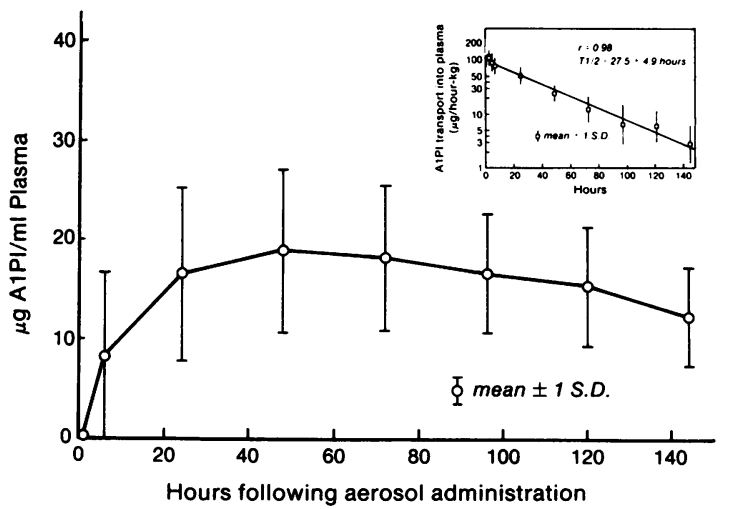

Figure 5. Levels of human A1PI in group III dogs peak at $48 \mathrm{~h}$ after inhalation of human A1PI aerosol. The plasma A1PI levels were used to calculate rates of A1PI transport into the plasma. (Inset) The calculated A1PI transport rates closely fit a monoexponential equation with a $t_{1 / 2}$ of $27.5 \pm 4.9 \mathrm{~h}$. provides an estimate of the amount of A1PI transported out of the lung at any time after aerosol delivery:

$\mathrm{A} 1 \mathrm{PI}=4.054\left[1-\mathrm{e}^{-0.0252 t}\right] \mathrm{mg} \cdot \mathrm{kg}^{-1}$

The $t_{1 / 2}$ for the transport of A1PI out of the lung calculated from these equations is $\ln (2) / 0.0252=27.5 \pm 4.9 \mathrm{~h}$. The estimate for the total amount of A1PI that will pass into the plasma is $4.05 \mathrm{mg} / \mathrm{kg}$ or $15.3 \%$ of the amount nebulized. This amount of A1PI is similar to the amount predicted to be present in the lung periphery after aerosol A1PI administration, $4.22 \mathrm{mg} / \mathrm{kg}(26.4 \mathrm{mg} / \mathrm{kg}$ A1PI nebulizer chamber $\cdot 16 \%$ peripheral deposition) using the deposition data obtained from gamma camera scanning.

Passage of aerosolized A1PI into plasma and lymph of sheep. To examine the transport of A1PI across the epithelial and endothelial components of the alveolar-capillary membrane (ACM), we analyzed antigenic levels of A1PI in sheep lymph and plasma after aerosolization of A1PI (Fig. 6). The levels of A1PI measured in sheep lymph showed a rapid rise to a peak level of $37.2 \pm 42.0 \mu \mathrm{g} / \mathrm{ml}$ at $8 \mathrm{~h}$ following inhalation, then a decrease to levels similar to those in plasma by $120 \mathrm{~h}$. There was considerable variability in the peak lymph level of A1PI, which we attributed to possible differences in the amount of A1PI deposited in the lung of each sheep; because we did not have any measurement of the amount of A1PI deposited in the sheep lung, we did not use lymph levels of A1PI to model epithelial permeability.

The plasma levels of A1PI seen in sheep after inhalation of the A1PI aerosol changed in a manner similar to that seen in the dog studies, although the peak levels were somewhat less. The measured plasma levels of A1PI were analyzed in a manner similar to that performed in dogs. To correct for the A1PI lost in the lymph drained through the cannula, the rate of loss of A1PI through the cannulated lymph was calculated from the product of the lymph drainage rate and the human A1PI content. This rate of lymph A1PI drainage $(16.1 \pm 10.4 \%$ of the total rate of A1PI transport out of the lung) was added to the calculated A1PI transport into the plasma for each time point. The calculated total A1PI transport out of the lung was used to calculate the monoexponential equation:

$\frac{\mathrm{dA} 1 \mathrm{PI}}{\mathrm{d} t}=0.0564 \mathrm{e}^{-0.0185 t} \mathrm{mg} \cdot \mathrm{kg}^{-1} \cdot \mathrm{h}^{-1} \quad(r=0.86)$.

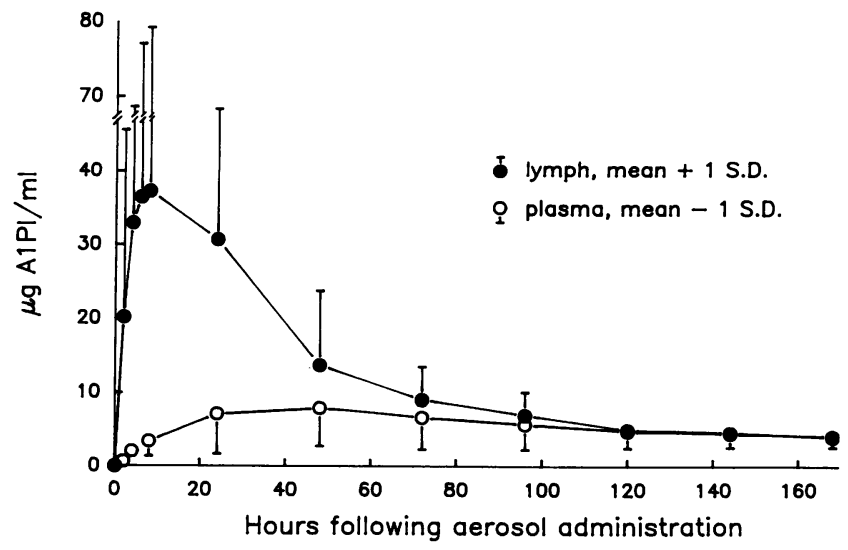

Figure 6. Levels of antigenic human A1PI in sheep plasma (open circles) and lymph (closed circles) following inhalation of human A1PI are plotted against time after inhalation. Values are expressed as mean $\pm S D$ of four experiments. 
The integral of this equation (from time $=0$ to time $=t$ ) describes the amount of A1PI transported into plasma as a function of time:

AlPI $=3.047\left[1-\mathrm{e}^{-0.0185 t}\right] \mathrm{mg} / \mathrm{kg}$

The exponent, $0.0185 \pm 0.0043$, provides an estimate of the $t_{1 / 2}$ of transport of A1PI into the sheep plasma of $37.5 \pm 7.1 \mathrm{~h}$. The amount of A1PI estimated from this equation to be present initially in the sheep lung after aerosolization, $3.05 \mathrm{mg} / \mathrm{kg}$, is slightly less than the value calculated for dogs, but this may reflect reduced efficiency of aerosol deposition; no tracer was used in the aerosol, so the amount of A1PI actually deposited in the lung initially was not measured directly.

Passage of intravenously administered ${ }^{125}$ I-AlPI into lymph of sheep. Intravenous injection of ${ }^{125} \mathrm{I}-\mathrm{A} 1 \mathrm{PI}$ into 4 sheep resulted in plasma radioactivity levels (Fig. 7) that could be modeled by a two-compartment, biexponential pharmacokinetic model (29). Plasma radioactivity fell with an initial $t_{1 / 2}$ of $4.80 \pm 2.0 \mathrm{~h}$ and a final steady-state $t_{1 / 2}$ of $74.4 \pm 5.6 \mathrm{~h}$. The volumes of distribution were $48.2 \pm 8.8$ and $109.4 \pm 16.0 \mathrm{ml} / \mathrm{kg}$ for the central and steady-state compartments, respectively. These values are not significantly different from those observed after injection of human A1PI into dogs: initial and final $t_{1 / 2}=5.54 \pm 0.77$ and $75.6 \pm 11.5 \mathrm{~h}$, respectively; central and steady-state volumes of distribution $=48.6 \pm 11.4$ and $97.9 \pm 18.7 \mathrm{ml} / \mathrm{kg}$ body weight, respectively (14). Plasma clearance of radioiodinated A1PI in these instrumented sheep was $1.10 \pm 0.14 \mathrm{ml} \cdot \mathrm{kg}^{-1} \cdot \mathrm{h}^{-1}$. The calculated pharmacokinetic parameters for individual animals were used for the subsequent analysis of plasma and lymph data.

The levels of radioactivity in lung lymph rose rapidly after intravenous injection of ${ }^{125} \mathrm{I}$-A IPI and, after equilibrium was reached, fell in parallel with plasma levels (Fig. 6). Of the injected radioactivity, $0.71 \%$ was recovered from the lung lymph collection over the course of the study, an amount too small to effect the calculation of the kinetic parameters following intravenous injection.

To estimate the permeability of the endothelial membrane of sheep for A1PI, the $t_{1 / 2}$ for transport of A1PI across the

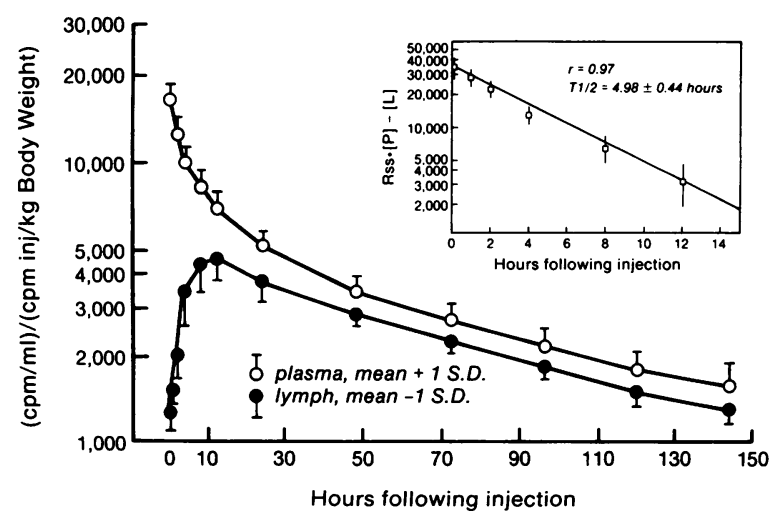

Figure 7. Sheep plasma (open circles) and lymph (closed circles) radioactivity per milliliter are shown on a semilog scale plotted against time after injection of $100 \mu \mathrm{Ci}$ of ${ }^{125} \mathrm{I}-\mathrm{A} 1 \mathrm{PI}$. Values are normalized to the injected radioactivity and body weight and are shown as the mean $\pm S D(n=4)$. The steady-state ratio, $R_{\mathrm{ss}}$, between lymph and plasma radioactivity, $0.88 \pm 0.086$, is used to calculate the quantity $R_{\mathrm{ss}} \cdot[P]-[L]$. This quantity describes the equilibration of A1PI across the endothelium and is shown in the inset plotted against time after injection of the A1PI. The calculated values fit a monoexponential equation with a $t_{1 / 2}$ of $4.98 \pm 0.44 \mathrm{~h}$. endothelium was calculated by performing a nonlinear least squares regression on the radioactivity levels measured in plasma and lymph using the relationship:

$\left(\mathrm{R}_{\mathrm{ss}} \cdot P\right)-L=C \mathrm{e}^{-b t}$

where $R_{s s}, 0.822 \pm 0.086$, is the steady-state ratio between lymph and plasma ${ }^{125} \mathrm{I}-\mathrm{A} 1 \mathrm{PI}$ measured in simultaneous plasma and lymph samples obtained $>24 \mathrm{~h}$ after the injection of the ${ }^{125} \mathrm{I}-\mathrm{A} 1 \mathrm{PI}$, and $P$ and $L$ are the respective levels of radioactivity per milliliter of plasma and lymph. Using this approach (Fig. 6, inset), the $t_{1 / 2}$ for transport of A1PI across the endothelium is $4.98 \pm 0.44 \mathrm{~h}(b=0.139$, average $r=0.96)$.

Estimates of permeabilities to A1PI. Using the values we measured for the $t_{1 / 2}$ of transport of A1PI across the endothelium and the $t_{1 / 2}$ for transport across the entire alveolar-capillary membrane and using two independent methods, we have calculated the permeability of the components of the alveolarcapillary membrane to A1PI.

First, the $t_{1 / 2}$ for transport of a marker substance across a membrane and the permeability of that membrane for a marker substance can be used with the $t_{1 / 2}$ for transport of A1PI across the same membrane to calculate the permeability of that membrane for A1PI using the relationship:

Permeability $_{\mathrm{AIPI}}=$ Permeability $_{\text {Marker }} \cdot \frac{t_{1 / 2 \text { Marker }}}{t_{1 / 2 \mathrm{~A} I \mathrm{PI}}}$

Gorin and Stewart (32) measured the $t_{1 / 2}$ of transport and permeability of infused radioiodinated albumin across the endothelium and across the ACM into the alveolus in sheep instrumented in a manner identical to that in our experiments. These values $\left(t_{1 / 2}\right.$ of transport $=4.0$ and $8.1 \mathrm{~h}$, permeability $=2.92 \times 10^{-8}$ and $2.13 \times 10^{-9} \mathrm{~cm} / \mathrm{s}$ for endothelium and ACM, respectively) were used to calculate the permeability of the endothelium and the ACM of sheep to A1PI (Table III, method $A$ ).

Alternatively, permeability of A1PI across a barrier can be calculated directly from the $t_{1 / 2}$ of transport across that barrier using the relationship:

Permeability $=\frac{0.693 \cdot V_{\mathrm{A}} \cdot V_{\mathrm{B}}}{T_{\mathrm{Barrier}} \cdot S \cdot\left(V_{\mathrm{A}}+V_{\mathrm{B}}\right)}$

where $S$ is the surface area of the barrier, $V_{\mathrm{A}}$ and $V_{\mathrm{B}}$ are the volumes occupied by A1PI on each side of the barrier, and $T_{\text {Barrier }}$ is the $t_{1 / 2}$ for transport of A1PI across the barrier. For calculation of permeability of A1PI across dog or sheep ACM, $0.060 \mathrm{ml} / \mathrm{g}$ wet lung weight (34) was used to estimate the alveolar fluid volume, $V_{\mathrm{A}}$. The steady-state volume of distribution of A1PI, $99.9 \mathrm{ml} / \mathrm{kg}$ body weight for dogs (14) and $109 \pm 16 \mathrm{ml} / \mathrm{kg}$ for sheep, was used for $V_{\mathrm{B}}$. A value of 661.6 $\mathrm{cm}^{2} / \mathrm{g}$ wet lung weight (32) was used for the alveolar surface area, $S$. For calculation of the permeability of A1PI across the sheep endothelium, we used $0.197 \mathrm{ml} / \mathrm{g}$ wet lung weight (35) as the interstitial volume, $V_{\mathrm{A}}$. The plasma volume, calculated as the initial volume of distribution of A1PI of each sheep $48.2 \pm 8.8 \mathrm{ml} / \mathrm{kg}$, was used for $V_{\mathrm{B}}$. A wet lung weight of 595 $\mathrm{cm}^{2} / \mathrm{g}$ was used as the capillary surface area based on a ratio of capillary to alveolar surface area of 0.9 (36). The results of these calculations, using the $t_{1 / 2}$ values for transport of A1PI across the ACM presented in this paper, are shown in Table III, method $B$.

The permeability of the epithelium to A1PI is obtained by assuming the resistances to passage of A1PI across the alveolar 
Table III. Calculated Permeabilities

\begin{tabular}{|c|c|c|c|c|c|c|}
\hline \multirow[b]{3}{*}{ Animal } & \multicolumn{3}{|c|}{ Alveolar-capillary membrane } & \multicolumn{3}{|c|}{ Endothelium } \\
\hline & \multirow[b]{2}{*}{$t_{1 / 2}$} & \multicolumn{2}{|c|}{ AIPI permeability } & \multirow[b]{2}{*}{$t_{1 / 2}$} & \multicolumn{2}{|c|}{ AIPI permeability } \\
\hline & & Method $A$ & Method $B$ & & Method $A$ & Method $B$ \\
\hline & $h$ & $\mathrm{~cm} / \mathrm{s}$ & $10^{-10}$ & $h$ & $\mathrm{~cm} / \mathrm{s}$ & $10^{-10}$ \\
\hline \multirow[t]{3}{*}{ Dog } & $50.8^{*}$ & 一 & $3.49 \pm 0.51$ & - & & \\
\hline & $27.5^{\ddagger}$ & - & $6.39 \pm 0.83$ & - & & \\
\hline & $30.8^{\S}$ & - & 5.64 & - & & \\
\hline Sheep & $37.5^{\ddagger}$ & $4.61 \pm 0.93$ & $4.63 \pm 0.94$ & $4.98^{\prime \prime}$ & $236 \pm 17.3$ & $122 \pm 9.2$ \\
\hline
\end{tabular}

Values for the permeability of the alveolar-capillary membrane or the pulmonary endothelium to AIPI were calculated as described in the text using either Eq. 2, method $A$, or Eq. 3, method $B$. The values for $t_{1 / 2}$ of transport of A1PI across the membrane were obtained from ${ }^{*}$ gamma camera scanning; ${ }^{\ddagger}$ measurement of plasma A I PI levels and calculation of transmembrane of A1PI flux; ${ }^{\S}$ measurement of the rate of equilibration between plasma and lavage A1PI levels after A1PI infusion in dogs (14); or "measurement of the rate of equilibration between lymph and plasma of ${ }^{125}$ I-A 1 PI after intravenous injection.

capillary membrane are in series and therefore:

$$
\frac{1}{P_{\mathrm{ACM}}}=\frac{1}{P_{\mathrm{ENDO}}}+\frac{1}{P_{\mathrm{EPITH}}}
$$

Using the values for endothelial and ACM permeability obtained in sheep (Table III), we calculate the epithelial permeability to A1PI in sheep to be 4.70 or $4.81 \times 10^{-10} \mathrm{~cm} / \mathrm{s}$ from the values obtained with methods $A$ or $B$, respectively.

Organ distribution of inhaled ${ }^{131} I-A 1 P I$. The counts per gram of peripheral lung tissue obtained from dogs 6,24 , and $144 \mathrm{~h}$ after aerosolization of A1PI were multiplied by the wet weight of the excised lung to calculate total organ radioactivity. Total organ radioactivity, as a percent of the material initially aerosolized, was $10.7 \pm 8.5,6.0 \pm 4.4$, and $0.4 \pm 0.2 \%$ at 6 , 24, and $144 \mathrm{~h}$ after A1PI aerosolization, respectively. This reduction in lung radioactivity with time paralleled the decrease in external counts as measured by gamma camera scanning, $r=0.99$. If the curve of the decrease in whole lung radioactivity is extrapolated back to time $=0$, the total lung radioactivity immediately after aerosolization is found to $11.4 \pm 9 \%$ of the radioactivity initially aerosolized. This value is similar to the value of $16 \%$ that is predicted by external gamma camera scanning.

\section{Discussion}

The absence of adequate protease inhibitors in the lung is felt to contribute to the development of emphysema. Studies of parenteral administration of A1PI to deficient individuals in an effort to augment lung protease defenses are already in progress. Parenteral replacement is hampered by the need to maintain an elevation in total body A1PI levels although only a small portion of total body A1PI is resident in the lung. Because the lung appears to be the only site where lack of elastase inhibition results in disease, much of the intravenously administered A1PI may not be providing a useful function. Administration of A1PI as an aerosol presents the potential for direct and efficient augmentation of protease protection at the major target organ for proteolytic attack and provides an excellent model for increasing our understanding of protein transport in the lung.

Therapeutic use of A1PI aerosols requires: $(a)$ production of an aerosol that will penetrate to and deposit at the site of proteolytic attack; $(b)$ demonstration of retained antiprotease activity after deposition; and (c) knowledge of the kinetics of passage of A1PI from the lung and estimates of the levels of A1PI achieved in the alveolar, interstitial, and vascular compartments.

In this investigation, an ultrasonic nebulizer was used to produce a heterodisperse aerosol of A1PI that retained the antielastase properties of the starting material. The mass median aerodynamic diameter of this aerosol, $5.1 \mu \mathrm{M}$, is somewhat larger than has been considered ideal for maximal alveolar deposition in humans, but reduced efficiency of deposition may have been partially compensated for by use of slow inspiratory flow rates (29). Use of an ultrasonic nebulizer was also advantageous because of the large number of particles produced per unit volume and because there was no need for an auxiliary air source, thus allowing us to isolate the respiratory circuit containing radioactive aerosol particles.

The pattern of central deposition typical for most aerosolized medications is inappropriate for therapeutic aerosols of A1PI because the major site of proteolytic attack, the area where A1PI must be delivered, appears to be at the level of the alveolus or the respiratory bronchiole (38). Therefore, our first efforts centered on confirming that the A1PI aerosols produced in this study did deposit on the alveolar surface. First, immunohistologic staining allowed us to demonstrate that A1PI did penetrate into, and in fact coat, the surface of distal alveoli. Second, modeling loss of aerosolized A1PI from the lung was best accomplished with a biexponential equation. This finding is expected with simultaneous alveolar and proximal airway deposition when clearance rates from those two sites are different. Alveolar and proximal airway clearance rates can then be quantified using the two components of the biexponential model. We attribute the component of the equation with the shorter $t_{1 / 2}$ to A1PI deposited in the tracheobronchial tree, and the component with a longer $t_{1 / 2}$ to AlPI deposited in the alveolar region. There was also significant deposition of A1PI on more proximal airways, and we cannot exclude the possibility that some of the A1PI reached the alveolus by spreading from a more proximal site of deposition.

Analysis of the gamma scan data using a biexponential equation allowed us to estimate that $16 \%$ of the A1PI initially aerosolized into the airway was deposited in the alveolar region of the lung. This estimate for alveolar deposition of A1PI is supported by the examination of the radioactivity in the periphery of the lung at the time of each animal's killing. Our assumption that the component of the externally measured radioactivity with a slower clearance rate represents material deposited in alveoli is also supported by the analysis of the decline in radioactivity measured in concentric lung ROIs. Radioactivity measured over the central areas of the lung, those with a greater proportion of large airways, decreases more rapidly. Finally, the total amount of antigenic A1 PI, that we calculate to have passed through the plasma, based on measured plasma levels, is similar to the amount we predict to have deposited into the alveoli.

Lung lavage fluid obtained from dogs at intervals after inhalation of A1PI aerosols demonstrated increases in elastase 
inhibitory activity in proportion to the presence of human A1PI. The specific activity of the A1PI recovered in BAL was only modestly decreased from that of the starting A1PI preparation, indicating that aerosolization and deposition do not markedly alter A1PI activity. A1PI in lavage fluid retained its expected electrophoretic mobility and showed only scant evidence of proteolytic degradation.

Somewhat different $t_{1 / 2}$ values for transport of A1PI across the ACM of dogs were observed depending on the method of determination of the $t_{1 / 2}$. These values, 27.5 and $50.8 \mathrm{~h}$, result in ACM permeability values of $3.49 \pm 0.51$ and $6.39 \pm 0.83$ $\times 10^{-10} \mathrm{~cm} / \mathrm{s}$ (Table III). The difference between these values may be due to experimental variability or to unrecognized biases introduced by the different techniques used to obtain the estimates.

The values we obtained in these experiments for the $t_{1 / 2}$ for transport of A1PI out of the alveolus across the alveolar capillary membrane (ACM) are similar to our prior observation of A1PI transport into the alveolus after intravenous infusion in dogs (14). The $t_{1 / 2}$ for equilibration of A1PI levels between lavage fluid and plasma in that study, $44 \mathrm{~h}$, can be used with the known $t_{1 / 2}$ of A1PI in plasma over the period of observation, $103 \mathrm{~h}$, to calculate the $t_{1 / 2}$ for the transport of A1PI into the alveolus across the ACM using the approach of Gorin and Stewart (31). This value for the $t_{1 / 2}$ of transport of A1PI into the alveolus, $1 /(1 / 44+1 / 103)=30.8 \mathrm{~h}$, is in good agreement with the observations made in this study of the $t_{1 / 2}$ for transport of A1PI out of the alveolus in dogs and sheep (Table III). The similarity of these values is consistent with the transport process being a passive and symmetrical one.

The calculation of permeability of the endothelium and epithelium to A1PI demonstrate that the resistance to passage of A1PI across the alveolar capillary membrane is predominantly at the epithelium. The ratio of epithelial to total ACM resistance to A1PI transport is $0.96-0.98$, depending on the method of calculation. This ratio is similar to that suggested by Theodore et al. (37) for inulin (0.95) and to that calculated by Gorin and Stewart (31) for albumin (0.92). These calculations, as well as the measured levels of antigenic A1PI in sheep lung lymph, demonstrate that the steady-state levels of A1PI in the interstitium following inhalation of A1PI aerosols will be closer to levels of A1PI in plasma than to levels in the alveolus. The absolute magnitude of the A1PI levels in resident alveolar lining fluid are difficult to estimate because of the dilution induced by the process of performing BAL. Using the values we measured for peripheral deposition of radiolabeled A1PI and our estimates for alveolar lining fluid volume, the concentration of A1PI in alveolar lining fluid immediately after aerosolization is predicted to be $7.61 \mathrm{mg} / \mathrm{ml}$. This value is similar to the value we measured in BAL fluid $6 \mathrm{~h}$ after A1PI delivery if the level of albumin in alveolar lining fluid is assumed to be $30 \%$ of the level in plasma; alveolar lining fluid albumin levels have been variously estimated to range from 8.8 to $100 \%$ of plasma albumin levels $(32,39)$. Even assuming a minimum level of albumin in the alveolar lining fluid, the level of A1PI in the alveolar lining fluid at $6 \mathrm{~h}$ is $\sim 2.0 \mathrm{mg} / \mathrm{ml}$, or 40 times the peak level measured in lymph.

Because the elastin fibrils are within the interstitium, the implication that our results may have for therapeutic use of A1PI aerosols is not clear, and will depend on studies of the permeability of the epithelium to A1PI in disease states, on identification of critical sites of antiprotease deficiency, and on studies of the use of A1PI aerosols in models of lung injury. It may be that A1PI deposited in the alveolus can act as a reservoir and will be able to gain access to the interstitium in greater amounts at sites of active inflammation where permeability may be enhanced.

It is useful to note that, with the demonstration of alveolar region deposition, there was no alteration in arterial blood gases after exposure to the A1PI aerosol. This suggests that the administration and deposition of A1PI did not acutely alter $V / Q$ relationships, assuming that extrapulmonary factors $\left(\mathrm{P}_{\mathrm{I}} \mathrm{O}_{2}, \dot{V}_{\mathrm{A}}, \dot{V} \mathrm{O}_{2}\right.$, and $\left.Q_{\mathrm{T}}\right)$ did not change.

If aerosols of A1PI are used therapeutically, the values for transport of A1PI across the ACM can be used to estimate the steady-state levels of A1PI in alveolar fluid and plasma. In the steady state, the amount of A1PI cleared from the plasma must equal the amount of A1PI transported into the plasma from the lung. Therefore:

$$
P \cdot S_{\mathrm{A} I \mathrm{PI}-\mathrm{ACM}} \times[\mathrm{A} 1 \mathrm{PI}]_{\mathrm{Alv}}=\mathrm{Cl}_{\text {Plasma }} \times[\mathrm{A} 1 \mathrm{PI}]_{\mathrm{Plasma}}
$$

or

$$
\frac{[\mathrm{A} 1 \mathrm{PI}]_{\mathrm{Plasma}}}{[\mathrm{A} 1 \mathrm{PI}]_{\mathrm{Alv}}}=\frac{(P \cdot S)_{\mathrm{AIPI}-\mathrm{ACM}}}{\mathrm{Cl}_{\mathrm{Plasma}}}
$$

If we assume an ACM permeability to AIPI in humans of $4.6 \times 10^{-10} \mathrm{~cm} / \mathrm{s}$, and an alveolar surface area of $70 \mathrm{~m}^{2}(35)$, then the $(P \cdot S)_{\mathrm{AIPI}-\mathrm{ACM}}$, the permeability-surface area product, will be $3.22 \times 10^{-4} \mathrm{~cm}^{3} / \mathrm{s}$. Plasma clearance of A1PI in man is $0.7 \mathrm{ml} \cdot \mathrm{kg}^{-1} \cdot \mathrm{h}^{-1}$ or $1.36 \times 10^{-2} \mathrm{ml} / \mathrm{s}$ (unpublished data) and so the expected ratio of A1PI levels between plasma and alveolar fluid is 0.024 . If alveolar levels of A1PI are maintained at $1.0 \mathrm{mg} / \mathrm{ml}, 5$-fold above normal levels and 15 -fold greater than the predicted protective level of A1PI in the alveolus (13), then the transalveolar flux of A1PI will be $28 \mathrm{mg} / \mathrm{d}$ and plasma levels of the inhaled A1PI will be $0.024 \mathrm{mg} / \mathrm{ml}$. Given an efficiency of $16 \%$ for the delivery of aerosolized A1PI into the alveolus, $175 \mathrm{mg}$ of fully active A1PI will need to be aerosolized per day, or $1225 \mathrm{mg} / \mathrm{wk}$, to maintain this level of A1PI in the alveolar fluid. This amount of A1PI is 30\% of the 4,200 $\mathrm{mg} / \mathrm{wk}$ of the same A1PI preparation that is required for parenteral replacement therapy using a dose of $60 \mathrm{mg} \cdot \mathrm{kg}^{-1}$ per wk in a 70-kg subject (13). Using the same preparation of A1PI with the equipment described in this study, this amount of A1PI would represent a volume of $\sim 9 \mathrm{ml}$ before dilution. Improved aerosol delivery methods may further reduce the amount of A1PI required.

In summary, we have demonstrated that aerosols of A1PI can be produced that retain their antielastase activity and can penetrate into and deposit on the surface of the distal airspaces of the lung. AlPI recovered from BAL as late as $144 \mathrm{~h}$ after inhalation of the aerosol demonstrates persistent antielastase activity. Estimates of the permeability of the endothelium and epithelium to A1PI suggest that, in the intact uninflamed lung, the major barrier to A1PI transport is at the epithelium. Use of A1PI aerosols will maintain protective alveolar levels of A1PI despite levels of plasma A1PI that would normally be considered subtherapeutic.

\section{Acknowledgments}

We would like to thank Ronald Konopka, M. Terry Hartman, and James Marsh for excellent technical assistance; Dr. Peter D. Wagner for review of the manuscript; and Dr. Kenneth M. Moser for his helpful discussion.

This work was supported in part by grants GM-33324, HL-23584, HL-34752, and HL-36279 from the National Institutes of Health. 
Note added in proof: During review of this paper, Hubbard et al. submitted evidence that recombinant DNA-produced A1PI aerosolized to sheep retained biologic activity but was not detectable in lymph, blood, or lung lavage after $36 \mathrm{~h}(40)$.

\section{References}

1. Janoff, A. 1985. Elastases and emphysema. Current assessment of the protease-antiprotease hypothesis. Am. Rev. Respir. Dis. 132:417-433.

2. Mass, B., T. Ikeda, D. R. Meranze, G. Weinbaum, and P. Kimbel. 1972. Induction of experimental emphysema. Cellular and species specificity. Am. Rev. Respir. Dis. 106:384-391.

3. Senior, R. M., H. Tegner, C. Kuhn, K. Ohlsson, B. C. Starcher, and J. A. Pierce. 1977. The induction of pulmonary emphysema with human leukocyte elastase. Am. Rev. Respir. Dis. 116:469-475.

4. Kidikoro, Y., T. C. Kravis, K. M. Moser, J. C. Taylor, and I. P. Crawford. 1977. Relationship of leukocyte elastase concentration to severity of emphysema in homozygous alpha ${ }_{1}$-antitrypsin-deficient persons. Am. Rev. Respir. Dis. 115:793-803.

5. Gadek, J. E., G. A. Fells, R. L. Zimmerman, S. I. Rennard, and R. G. Crystal. 1981. Antielastases of the human alveolar structures. Implications for the protease-antiprotase theory of emphysema. $J$. Clin. Invest. 68:889-898.

6. Larsson, C. 1978. Natural history and life expectancy in severe alpha $a_{1}$-antitrypsin deficiency, PiZ. Acta. Med. Scand. 204:345-351.

7. Black, L. F., and F. Kueppers. 1978. Alpha ${ }_{1}$-antitrypsin deficiency in nonsmokers. Am. Rev. Respir. Dis. 117:421-428.

8. Eriksson, S. 1964. Pulmonary emphysema and alpha ${ }_{1}$-antitrypsin deficiency. Acta. Med. Scand. 177:175-179.

9. Carp, H., F. Miller, R. Hoidal, and A. Janoff. 1982. Potential mechanism of emphysema: alpha ${ }_{1}$-proteinase inhibitor recovered from lungs of smokers contains oxidized methionine and has decreased elastase inhibitory capacity. Proc. Natl. Acad. Sci. USA. 79:2041-2045.

10. Kaplan, P. D., C. Kuhn, and J. A. Pierce. 1973. The induction of emphysema with elastase. 1. The evolution of the lesion and the influence of serum. J. Lab. Clin. Med. 82:349-356.

11. Martorana, P. A., and N. N. Share. 1976. Effect of human alpha ${ }_{1}$-antitrypsin on papain-induced emphysema in the hamster. $\mathrm{Am}$. Rev. Respir. Dis. 113:607-612.

12. Gadek, J. E., H. G. Klein, P. V. Holland, and R. G. Crystal. 1981. Replacement therapy of alpha ${ }_{1}$-antitrypsin deficiency. Reversal of protease-antiprotease imbalance within the alveolar structures of PiZ subjects. J. Clin. Invest 68:1158-1165.

13. Wewers, M. D., A. Casolaro, S. E. Sellers, S. C. Swayze, K. M. McPhaul, and R. G. Crystal. 1987. Replacement therapy for alpha $1^{-}$ antitrypsin deficiency associated with emphysema. N. Engl. J. Med 316:1055-1062.

14. Smith, R. S., R. G. Spragg, K. M. Moser, C. G. Cochrane, and J. P. McCarren. 1987. Pulmonary penetration of alpha proteinase $_{1}$ inhibitor administered parenterally to dogs. Am. Rev. Respir. Dis. 137:1391-1398.

15. Coan, M. H., W. J. Brockway, H. Equizabal, T. Kreig, and M.

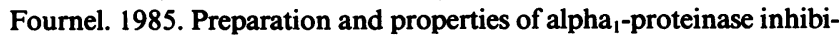
tor concentrate from human plasma. Vox Sang. 48:333-342.

16. Pannell, R., D. Johnson, and J. Travis. 1974. Isolation and properties of human plasma alpha-1-proteinase inhibitor. Biochemistry. 13:5439-5445.

17. Laemmli, U. K. 1970. Cleavage of structural proteins during the assembly of the head of bacteriophage $\mathrm{T}_{4}$. Nature (Lond.). 227:680-685.
18. David, G. S., and R. A. Reisfeld. 1974. Protein iodination with solid state lactoperoxidase. Biochemistry. 13:1014-1021.

19. Laurell, C. B. 1972. Electroimmunoassay. Scand. J. Clin. Lab. Invest. 29(Suppl. 124):21-23.

20. Jeppson, J. O., C. B. Laurell, and M. Fagerhol. 1978. Properties of isolated human alpha-1-antitrypsins of Pi types M, S, and Z. Eur. J. Biochem. 83:143-153.

21. Bieth, J., B. Spiess, and C. G. Wermuth. 1974. Synthesis and analytical use of a highly sensitive and convenient substrate of elastase. Biochem. Med. 11:350-357.

22. Phalen, R. F. 1984. Inhalation studies: foundations and techniques. CRC Press, Boca Raton, FL. 115-120.

23. Smith, R. M., and R. G. Spragg. 1988. Aerosol administration of alpha-1-proteinase inhibitor to dogs. Am. J. Med. 84:48-52.

24. Hsu, S. M., L. Raine, and H. Fanger. 1981. The use of avidinbiotin-peroxidase complex (ABC) in immunoperoxidase techniques: a comparison between $\mathrm{ABC}$ and antibody (PAP) procedures. J. Histochem. Cytochem. 29:577-586.

25. Staub, N., R. Bland, K. Brigham, R. Demling, A. V. Erdman III, and C. W. Woolverton. 1975. Preparation of chronic lung lymph fistulas in sheep. J. Surg. Res. 19:315-322.

26. Snedecor, G. W., and W. G. Cochran. 1980. Statistical Methods. 7th ed. Iowa State University Press, Ames, IA. 166.

27. Sedman, A. J., and J. G. Wagner. 1976. CSTRIP, a Fortran IV computer program for obtaining initial polyexponential parameter estimates. J. Pharmacol. Sci. 65:1006-1010.

28. Metzler, C., G. Elfring, and A. McEwen. 1974. A Users Manual for NONLIN and Associated Programs. The Upjohn Co., Kalamazoo, MI.

29. Gibaldi, M., and D. Panier. 1982. Pharmacokinetics. 2nd ed. Marcel Dekker, Inc., New York, NY.

30. Schlesinger, R. B. 1985. Comparative deposition of inhaled aerosols in experimental animals and humans: a review. J. Toxicol. Environ. Health. 15:197-214.

31. Taylor, A. E., A. C. Guyton, and V. S. Bishop. 1965. Permeability of the alevolar membrane to solutes. Circ. Res. XVI:353-362.

32. Gorin, A. B., and P. A. Stewart. 1979. Differential permeability of endothelial and epithelial barriers to albumin flux. J. Appl. Physiol. 47:1315-1324.

33. Wangersteen, O. D., L. E. Wittmers, Jr., and J. A. Johnson. 1969. Permeability of the mammalian blood-gas barrier and its components. Am. J. Physiol. 216:719-727.

34. Guyton, A. C., A. E. Taylor, R. E. Drake, and X. Parker. 1976. Dynamics of subatmospheric particles in the pulmonary interstitium. In Lung Liquids. Ciba Foundation, London, UK. 38:77-96.

35. Selinger, S. L., R. D. Bland, R. H. Demling, and N. C. Staub. 1975. Distribution volumes of $\left[{ }^{131} \mathrm{I}\right]$ albumin, $\left[{ }^{14} \mathrm{C}\right]$ sucrose, and ${ }^{36} \mathrm{Cl}$ in sheep lung. J. Appl. Physiol. 39:773-779.

36. Weibel, E. R. 1973. Morphocological basis of alveolar-capillary gas exchange. Physiol. Rev. 53:419-495.

37. Theodore, J., E. D. Robin, R. Gaudio, and J. Acevedo. 1975. Transalveolar transport of large polar solutes (sucrose, inulin, and dextran). Am. J. Physiol. 229:989-996.

38. Eriksson, S. 1965. Studies in alpha-1-antitrypsin deficiency. Acta Med. Scand. 177(Suppl 432):1-85.

39. Rennard, S. I., G. Basset, D. Lecossier, K. M. O'Donnell, P. Pinkston, P. G. Martin, and R. G. Crystal. 1986. Estimation of the volume of epithelial lining fluid recovered by lavage using urea as a marker of dilution. J. Appl. Physiol. 60:532-538.

40. Hubbard, R. C., M. A. Casolaro, M. Mitchell, S. E. Sellers, F. Arabia, M. A. Matthay, and R. G. Crystal. 1989. Fate of aerosolized recombinant DNA-produced $\alpha_{1}$-antitrypsin: use of the epithelial surface of the lower respiratory tract to administer proteins of therapeutic importance. Proc. Natl. Acad. Sci. USA. 86:680-684. 